\title{
PREVALÊNCIA E CORRELAÇÃO ENTRE OBESIDADE, HIPERTENSÃO ARTERIAL E A PRÁTICA DE ATIVIDADE FÍSICA
}

\author{
Natália Turri da Silva', Thais Roque Giacon¹, Marianne Penachini da Costa ${ }^{1}$, Ana Laura Ricci Vitor ${ }^{2}$, Luiz \\ Carlos Marques Vanderlei ${ }^{3}$ \\ ${ }^{1}$ Discente do Curso de Graduação em Fisioterapia, ${ }^{2}$ Discente do Curso de Pós-Graduação em Fisioterapia, ${ }^{3}$ Docente do Departamento \\ de Fisioterapia da Faculdade de Ciências e Tecnologia - FCT/UNESP, Presidente Prudente.
}

\section{RESUMO}

Nas últimas décadas, obesidade e sobrepeso, importantes fatores de risco para o desenvolvimento de doenças cardiovasculares, tiveram um aumento importante, sendo o sedentarismo uma de suas principais causas. Partindo disso, o objetivo deste trabalho foi analisar a prevalência da obesidade e HA de frequentadores do CEAFiR, além de correlacionar valores de IMC com valores de PAS e PAD. Para isso foram coletados peso e altura, para obtenção do IMC, e PA de 163 indivíduos (114 mulheres e 49 homens) com média de idade de 53,0 $\pm 16,1$ anos. Os resultados mostraram que $30,1 \%$ possuem obesidade e $43,6 \%$ sobrepeso. Em relação à $\mathrm{HA}, 22,7 \%$ encontram-se nos estágios de hipertensão 1, 2 ou 3 e 7,4\% hipertensão sistólica isolada. Portanto, 73,7\% estão acima do peso e 30,1\% são hipertensos. Este resultado reforça a importância de programas de prevenção que visam orientar a população, promovendo modificações positivas no estilo de vida.

Palavras-chave: obesidade, hipertensão arterial, doenças cardiovasculares.

\section{PREVALENCE AND CORRELATION AMONG OBESITY, HYPERTENSION AND THE PHYSICAL ACTIVITY PRACTICE}

\begin{abstract}
In the recent decades, obesity and overweight, important risk factors for the cardiovascular disease development, had a significant increase being the sedentarism one of the most important causes. Considering it, this study aims at analysing the prevalence of obesity and hypertension in users of CEAFiR, besides correlating BMI with SBP and DBP. For that, weight and height were collected to calculate BMI and BP of 163 subjects (114 women and 49 men) whose average age was $53.0 \pm 16.1$ years old. The results showed that $30.1 \%$ were classified as obese and $43.6 \%$ as overweight. Regarding to $\mathrm{HA}, 22,7 \%$ were classified in 1, 2 or 3 stages of hypertension and $7.4 \%$ as isolated systolic hypertension. Therefore, $73,7 \%$ were classified as overweight and $30.1 \%$ with hypertension. This result reinforces the importance of preventional programs aiming at orient the population, promoting positive changes in lifestyle.
\end{abstract}

Keywords: obesity, hypertension, cardiovascular disease. 


\section{INTRODUÇÃO}

Nas últimas décadas a obesidade e o sobrepeso, importantes fatores de risco para o desenvolvimento de doenças cardiovasculares, tiveram um aumento significativo, sendo $\mathrm{O}$ sedentarismo uma de suas principais causas ${ }^{(1,2)}$.

Quando comparados, indivíduos de peso normal em relação a obesos e sobrepeso, estes possuem maior risco de desenvolver hipertensão arterial (HA). A HA é considerada um problema de saúde pública por sua magnitude, risco e dificuldades no controle. É uma afeç̧ão comum, assintomática, prontamente detectável, em geral de fácil tratamento e que costuma gerar complicações letais quando não tratada. Apresenta custos médicos e socioeconômicos elevados, decorrentes principalmente das suas complicações, como: doença cerebrovascular, doença arterial coronariana, insuficiência cardíaca, insuficiência renal crônica e doença vascular periférica ${ }^{(3)}$.

Outro fator de grande relevância é o sedentarismo, que além de ser fator causal para obesidade e sobrepeso também favorece $\mathrm{O}$ aparecimento de doenças do aparelho cardiocirculatório ${ }^{(2)}$.

As evidências acumuladas nos últimos anos afirmam que condutas não-medicamentosas devem ser a estratégia inicial para o tratamento de sobrepeso e hipertensão leve a moderada, sendo a prática de exercício físico regular uma alternativa relevante que promove redução da gordura corporal total e gordura subcutânea abdominal, controle da pressão arterial, além de melhora da resistência à insulina, efetuando importantes alterações autonômicas e hemodinâmicas que influenciam no desempenho do sistema cardiocirculatório ${ }^{(4,5)}$.

Em meio a esse contexto, pode-se inferir que a atividade física não promove apenas resultados benéficos para indivíduos já acometidos por patologias do aparelho cardiovascular e obesidade, mas também atua de forma preventiva para tais doenças ${ }^{(4,6,7)}$.

Algo a ser considerado ainda em relação à prática de atividade física regular é o fato de ser uma atitude simples e fácil que contribui para o controle da pressão arterial e do sobrepeso. O acompanhamento dos índices pressóricos pode mostrar uma redução nos valores de hipertensão arterial sistólica e diastólica, revelando a eficiência da prática ${ }^{(4)}$.

Além disso, estudos mostram que a prática regular de atividade física não promove apenas uma melhora do sistema cardiovascular, mas também do sistema digestivo, imunológico, nervoso, músculo esquelético e respiratório concomitantemente a melhora da auto-estima e alívio do estresse colaborando para uma melhora da qualidade de vida ${ }^{(8)}$.

\section{OBJETIVOS}

Sendo assim, este estudo teve por objetivo primeiro analisar a prevalência da obesidade e HA de frequentadores do Centro de Estudos e Atendimento em Fisioterapia e Reabilitação da FCT/Unesp (CEAFiR), além de correlacionar valores de índice de massa corporal (IMC) com valores de pressão arterial sistólica (PAS) e diastólica (PAD).

\section{METODOLOGIA}

O estudo foi realizado em Presidente Prudente, São Paulo - Brasil, com os indivíduos que participaram da Semana de prevenção contra a obesidade e a hipertensão arterial, vinculada ao projeto de extensão universitária intitulado "Programa de prevenção primária e secundária de doenças cardiovasculares do Centro de Estudos e Atendimento em Fisioterapia e Reabilitação CEAFiR - FCT/ UNESP".

A massa corporal foi obtida por meio da medida em balança da marca Welmy, com os indivíduos em posição ortostática com os braços 
estendidos ao longo do corpo, sem calçados. Para a obtenção da estatura, os voluntários encontravam-se também descalços em estadiômetro da marca Sanny. A partir das medidas de massa corporal e estatura, o índice de massa corporal (IMC) foi calculado utilizandose a massa do indivíduo (quilogramas), divida pela sua altura (metros) ao quadrado; a fim de se obter a classificação da obesidade. Os valores de IMC foram classificados em: IMC $\leq 18,5 \mathrm{~kg} / \mathrm{m}^{2}$ (baixo peso), IMC entre $18,5 \mathrm{~kg} / \mathrm{m}^{2}$ e $25 \mathrm{~kg} / \mathrm{m}^{2}$ (normal), IMC entre $25 \mathrm{~kg} / \mathrm{m}^{2}$ e $30 \mathrm{~kg} / \mathrm{m}^{2}$ (sobrepeso) e IMC $\geq 30 \mathrm{~kg} / \mathrm{m}^{2}$ (obeso), conforme as Diretrizes Brasileiras de Obesidade (2009/2010).

A pressão arterial sistólica (PAS) e a pressão arterial diastólica (PAD) (em $\mathrm{mmHg}$ ) foram obtidas de modo indireto com o uso de esfigmomanômetro anaeróide, e classificadas segundo a V Diretrizes Brasileira de Hipertensão Arterial (V Diretrizes de HA, 2007); foram classificados como hipertensos os indivíduos que apresentavam pressão sistólica $\geq 140 \mathrm{mmHG}$ e pressão diastólica $\geq 90 \mathrm{mmHg}$, ou aqueles que estavam em uso de medicação anti-hipertensiva; já quem apresentava pressão sistólica $\geq$ $120 \mathrm{mmHg}$ e pressão diastólica $\geq 80 \mathrm{mmHg}$ foi considerado com pressão abaixo da normal; pressão sistólica igual a $120 \mathrm{mmHg}$ e diastólica igual a $80 \mathrm{mmHg}$ caracterizou aqueles com pressão normal. A pressão arterial foi aferida apenas uma vez, antes do início da entrevista.

Para caracterização da amostra foi realizada estatística descritiva e, como os dados apresentaram distribuição normal, a correlação de Pearson foi utilizada; o nível de significância de $5 \%$ foi adotado para análise.

Além disso, foi realizada orientação dos indivíduos com distribuição de folder informativo e explicações com respaldo científico. O folder continha informações sobre os benefícios da atividade física como melhora de vários sistemas corporais como digestivo, imunológico, nervoso, músculo esquelético e respiratório, e também a respeito da melhora da auto-estima e alívio do estresse $^{(8)}$.

\section{RESULTADOS}

Foram analisados dados de 163 indivíduos (114 mulheres e 49 homens) com média de idade de 53,0 $\pm 16,1$ anos. Dos avaliados $30,1 \%$ (49) foram classificados como obesos, 43,6\% (71) com sobrepeso, 22,6\% (37) dentro da normalidade e 3,7\% (6) abaixo do peso. Em relação à $H A, 54,6 \%$ (89) apresentavam PA normal e ótima, 15,3\% (25) limítrofe, 22,7\% (37) nos estágios de hipertensão 1, 2, 3 e 7,4\% (12) hipertensão sistólica isolada. $\mathrm{Na}$ tabela abaixo (Tabela 1) são apresentadas as correlações encontradas.

Tabela 1. Correlação entre variáveis IMC, PAS e PAD.

\begin{tabular}{lcc}
\hline Variáveis & $\begin{array}{c}\text { Coeficiente de } \\
\text { Pearson }\end{array}$ & p valor \\
\hline IMC e PAS * & $r=0,205$ & 0,004 \\
IMC e PAD * & $r=0,226$ & 0,009 \\
\hline
\end{tabular}
* Correlações significantes com $\mathrm{p}<0,05$. IMC $=$ índice de
massa corporal. PAS $=$ pressão arterial sistólica. PAD=
pressão arterial diastólica.

\section{DISCUSSÃO}

O excesso de peso e a obesidade estão associados a uma série de fatores de risco para doenças, especialmente aquelas relacionadas ao sistema cardiovascular, sendo importante salientar que a mortalidade aumenta progressivamente com o aumento do $\mathrm{IMC}^{(6)}$.

No trabalho realizado, na Semana de prevenção contra a obesidade e a hipertensão arterial, encontrou-se que uma grande parte da população foi classificada como obesa ou com sobrepeso, havendo $73,7 \%$ de indivíduos com peso acima do desejável. E desta população analisada $30,1 \%$ foram classificados com 
hipertensão arterial no estágio 1,2 e 3 e hipertensão sistólica isolada. Além disso, houve correlação entre IMC e PAS e entre IMC e PAD, indicando que quanto maiores os valores de IMC maiores os valores de PAS e PAD.

Em estudo realizado em São Paulo, semelhante a diversos outros estudos que avaliam a influência da distribuição da gordura corporal sobre prevalência de HA e outros fatores de risco cardiovascular, verifica-se que indivíduos obesos têm maior predisposição para apresentar problemas cardiovasculares quando comparados a indivíduos de peso normal ${ }^{(3)}$.

A grande prevalência de HA em obesos tem sido atribuída à hiperinsulinemia (decorrente da resistência à insulina), principalmente em indivíduos que apresentam excesso de gordura na região abdominal. Este excesso de insulina provoca a ativação do sistema nervoso simpático e uma maior absorção do sódio, resultando com isso em aumento da resistência vascular periférica e da pressão arterial $^{(3)}$.

Diante disso, é necessária a intensificação de programas de controle de HA e outros fatores de risco cardiovasculares, visando uma diminuição do aparecimento de doenças cardiovasculares e melhor qualidade de vida à população. Uma das formas de realizar tal prevenção é por meio da atividade física, pois quando se avalia a associação entre atividade física e doenças cardiovasculares, observa-se que indivíduos sedentários apresentam maior probabilidade de desenvolver tais doenças ${ }^{(9)}$.

Atualmente, campanhas de combate ao sedentarismo recomendam a prática de trinta minutos de atividades físicas na maioria dos dias da semana envolvendo os grandes grupos musculares, podendo ser feita de forma contínua ou fracionada. Quando a prática esportiva é recomendada tem-se por objetivo quebrar a inércia para começar a adquirir hábitos de vida ativa e somente após algum tempo da prática regular, em ritmo moderado é que gradativamente vai se adquirindo os reais benefícios de proteção contra doenças cardiovasculares ${ }^{(9)}$.

Os resultados reforçam a importância de programas de prevenção de doenças cardiovasculares que visam orientação e a educação para produzir modificações positivas no estilo de vida dos indivíduos. Nesse sentido o estímulo à prática regular de atividade física funciona como uma alternativa satisfatória para controle de doenças, sendo resguardado 0 acompanhamento médico e de profissionais da saúde para indivíduos hipertensos.

\section{CONCLUSÃO}

Dos indivíduos avaliados, houve uma prevalência de $73,7 \%$ acima do peso desejável, $30,1 \%$ com hipertensão arterial estágios 1, 2 e 3 e com hipertensão arterial sistólica isolada. $E$ houve correlação entre IMC e PAS além de IMC e PAD mostrando que quanto maiores os valores de IMC, maiores os valores de PAS e PAD. A partir desses resultados ressalta-se a importância de programas de prevenção de doenças cardiovasculares e da presença de orientações a respeito da prática regular de atividade física, como alternativa de controle tanto de HA quanto da obesidade.

\section{REFERÊNCIAS}

1.Matsudo VKR, Matsudo SMM. Atividade Física no Tratamento de Obesidade. Einstein. 2006; (Supl 1):S29-S43.

2.Carneiro G, Faria NA, Filho FFR, Guimarães A, Lerário D, Ferreira RSG, Zanella MT. Influência da Distribuição da Gordura Corporal sobre A Prevalência da Hipertensão Arterial e Outros Fatores de Risco Cardiovascular em Indivíduos Obesos. Rev Assoc Med Bras. 2003;49(3):306-11 http://dx.doi.org/10.1590/S0104- 
3;Nascente FMN, Jardim PCBV, Peixoto MRGP, Monego ET, Barboso WKS, Moreira HG, et al. Hipertensão arterial e sua associação com índices antropométricos em adultos de uma cidade de pequeno porte do interior do Brasil. Rev Assoc Med Bras. 2009;55(6):716-722

4.Laterza MC, Rondon MUPB, Negrão CE. Efeito Anti-Hipertensivo do Exercício. Rev. Bras Hipertens. 2007;14(2):104-111.

5.Martin JFV, Cipullo JP. Tratamento Medicamentoso Para a Pré-hipertensão:para todos? Rev. Bras. Hipertens. 2009;16(1):44-45.

6.Nunes APOB, Rios ACS, Cunha GA, Barretto $A C P$, Negrão CE. Efeitos de um Programa de um Programa de Exercício físico Não Supervisionado e Acompanhado a Distância, Via Internet, sobre a Pressão Arterial e Composição Corporal em Indivíduos Normotensos e Pré-hipertensos. Arquivos Brasileiros de Cardiologia. 2006;86(4):289-296.

http://dx.doi.org/10.1590/S0066-

$\underline{782 \times 2006000400009}$

7.Monteiro MF, Filho DCS. Exercício físico e o controle da pressão arterial. Rev Bras Med Esporte. 2004, 10(6):513-516.

http://dx.doi.org/10.1590/S1517-

$\underline{86922004000600008}$

8.Stella F, Gobbi S, Corazza DI, Costa JLR. Depressão no Idoso: Diagnóstico, Tratamento e Benefícios da Atividade Física. Motriz. 2002;8(3):91-98.

9.Masson CRM, Dias-da-Costa JS, Olinto MTA, Meneghel S, Costa CC, Bairros F, et al. Prevalência de sedentarismo nas mulheres adultas da cidade de São Leopoldo, Rio Grande do Sul, Brasil. Cad. Saúde Pública. 2005; 21(6):1685-1694.

http://dx.doi.org/10.1590/S0102- 